\title{
Características biológicas e inmunológicas del veneno de Bothrops cotiara (Serpentes: Viperidae)
}

\author{
Adolfo Rafael de Roodt ${ }^{1}$, Judith Estévez ${ }^{2}$, Jorge Adrián Dolab ${ }^{1}$, Marcelo Víctor Manzanelli ${ }^{1}$, \\ Nicolás Piñeiro ${ }^{1}$, Jorge Francisco Paniagua ${ }^{3}$ \& Alejandro Urs Vogt ${ }^{4}$ \\ 1 Instituto Nacional de Producción de Biológicos, Administración Nacional de Laboratorios e Institutos de Salud "Dr. \\ Carlos G. Malbrán”, Ministerio de Salud. Av. Vélez Sarsfield 563, CP 1281, Buenos Aires, Argentina; \\ aderoodt@uolsinectis.com \\ 2 Instituto Bioclón S.A. de C.V., México DF, México. \\ 3 Laboratorios Silanes S.A. de C.V., México DF, México. \\ 4 Centro Zootoxicológico de Misiones, Oberá, Misiones, Argentina.
}

Recibido 18-III-2004. Corregido 16-VII-2004. Aceptado 31-X-2005.

\begin{abstract}
Biological and immunological characteristics of the poison of Bothrops cotiara (Serpentes: Viperidae). Bothrops cotiara is a venomous snake sporadically found in the province of Misiones in Argentina, South of Brazil and Paraguay. Data on the clinics of the envenomation produced by its bite and on its venom are scarce. There is no information on the neutralizing capacity of the antivenoms available. In this study, the lethal potency, hemorrhagic, necrotizing, coagulant and thrombin-like, defibrinogenating, indirect hemolytic and fibrinolytic activities of the venom of $B$. cotiara specimens from the province of Misiones were determined. The toxic activities were within the range of those described for the other Bothrops species from Argentina, and the electrophoretic and chromatographic studies showed similarities with those described for the other bothropic venoms. The immunochemical reactivity of six South American anti Viper antivenoms (ELISA) have a strong reactivity with all the antivenoms studied. The neutralizing capacity of three of these therapeutic antivenoms against the lethal potency and hemorrhagic, necrotizing, coagulant, thrombin-like and hemolytic activities showed a very close neutralizing capacity. Our data strongly suggest that the antivenoms for therapeutic use available in this area of South America are useful to neutralize the toxic and enzymatic activities of the venom of this uncommon specie of Bothrops. Rev. Biol. Trop. 54 (3): 889-901. Epub 2006 Sept. 29.
\end{abstract}

Key words: Snakes, Bothrops cotiara, toxicity, antivenom, neutralization.

Las diferentes especies del género Bothrops (Viperidae: Crotalinae) son responsables de la mayoría de los envenenamientos por mordeduras de serpientes venenosas en Latinoamérica (Costa Cardoso y Fan 1995, Russell et al. 1997, Alves Araujo et al. 2003). En la actualidad en la Argentina se comunican a las autoridades sanitarias alrededor 10000 accidentes anuales por animales venenosos -ponzoñosos(Ministerio de Salud 1999, Segre et al. 2000, García 2003). El 70 \% de estos accidentes son producidos por serpientes y de éstos, más del $95 \%$ son causados por especies del género Bothrops (Instituto Nacional de Producción de
Biológicos 1998, García 2003). En Brasil las mordeduras por Bothrops representan cerca del $90 \%$ de los casos de mordeduras por serpientes venenosas (Ministerio de Saude 1999, Alves Araujo et al. 2003) y en Centroamérica también las Bothrops son responsables de la mayoría de los accidentes (Rojas et al. 1997, Russell et al 1997, Sasa y Vazquez 2003).

Estas serpientes son conocidas popularmente en el Sur de Sudamérica (Paraguay, Sur de Brasil y Argentina) con el nombre de "yarará" (voz guaraní que da idea de “soberbia”) (Freiberg 1968, Bonnet y Pedace 1971), posiblemente debido a la firme actitud 
defensiva que presentan estos animales al sentirse amenazados.

En Argentina existen al menos siete especies de Bothrops. Seis de ellas se encuentran en la provincia de Misiones de las cuales cuatro pueden hallarse únicamente en esa provincia (Martino et al. 1979, Ministerio de Bienestar Social 1980, de Roodt y Troiano 1995) en la que se comparte la ofidiofauna con la de Paraguay y el Sur de Brasil. Entre las especies de Bothrops que allí pueden encontrarse está Bothrops (B.) cotiara (“cotiara”, "mboí-cotiá”, “yarará de panza negra”, “cotiarinha”). El hallazgo de esta serpiente es muy poco frecuente, aunque es constante a lo largo de los años. Lo reducido de su aparición hace pensar que podría estar en peligro de extinción, siendo que ya es muy difícil encontrar ejemplares en Brasil (Pessatti et al. 1995) y en la provincia de Misiones (Vogt 1985, Giraudo 2001).

Posiblemente a causa de lo anterior, el veneno de $B$. cotiara ha sido poco estudiado y solo algunos trabajos realizados con venenos provenientes de serpientes de Brasil han estudiado parcialemente sus actividades tóxicas (Ferreira et al. 1992, Dias Da Silva et al. 1989). Los trabajos disponibles sobre este veneno mencionan algunas de sus actividades biológicas como la trombina-símil (Nahas et al. 1979) que es muy importante en las crías (Furtado et al. 1991), una baja actividad sobre la agregación plaquetaria o su inhibición y su escasa actividad proteolítica (Francischetti et al. 1998). Se describió también que posee baja cantidad de componentes con actividad de fosfolipasa $A_{2}$ y poca actividad miotóxica (Moura da Silva et al. 1990, 1991) y una alta actividad de L-aminoácido-oxidasa (Pessatti et al. 1995). También se ha visto que posee una fracción altamente hemorrágica (Pessatti et al. 1995) la cual tiene alta reactividad inmunoquímica con la bothrojaracina de Bothrops jararaca (Castro et al. 1999). Sin embargo, no se dispone de datos de la toxicidad global del mismo así como de la neutralización de sus diferentes actividades tóxicas por antivenenos de uso terapéutico.

Debido a esta falta de conocimiento, el personal de salud requiere a menudo información que les indique cuál antiveneno deben aplicar ante su mordedura, dado que ninguno de los disponibles en Argentina, Uruguay o Brasil utilizan en sus mezclas inmunogénicas a este veneno. Por estos motivos estudiamos algunas de sus características bioquímicas y tóxicas, la reactividad inmunoquímica y la capacidad neutralizante de varios antivenenos de uso corriente en Sudamérica sobre el mismo.

\section{MATERIALES Y MÉTODOS}

Veneno: se utilizó veneno de ejemplares de $B$. cotiara adultos en buen estado de salud. Todos los ejemplares fueron hallados en la provincia de Misiones, Argentina y alojados en el Serpentario del Centro Zootoxicológico de Misiones (Oberá, Misiones) en cajas individuales con disponibilidad de agua ad libitum y con una alimentación de uno a cinco ratones semanales. El veneno fue extraído manualmente e inmediatamente desecado al vacío, tras lo cual se guardó a $-20{ }^{\circ} \mathrm{C}$ hasta su uso.

Determinación de la Potencia Letal: ratones de la cepa CF-1, de 18-20 g de peso se inyectaron ( $n=8$ por nivel de dosis) con diferentes dosis de veneno disuelto en $\mathrm{NaCl} 0.15$ M por la vía intraperitoneal (ip). A las 48 h se determinó la cantidad de animales muertos y la Dosis Letal 50 \% ( $\left(\mathrm{DL}_{50}\right)$ se estimó mediante el análisis por regresión no lineal de la curva dosis -porcentaje de mortalidad-, mediante el Software Prism versión 3 (Graph Pad Software, Inc. San Diego, California, USA). $\mathrm{La} \mathrm{DL}_{50}$ se definió como la dosis de veneno que produjo la muerte del $50 \%$ de los ratones dentro de un período de $48 \mathrm{~h}$.

SDS-PAGE: se realizó utilizando el sistema discontinuo descrito por Laemmli (1970). Brevemente, muestras del veneno entero o de sus fracciones cromatográficas se prepararon con dodecil sulfato de sodio (SDS) en condiciones no reductoras y se corrieron en geles homogéneos al $12.5 \%$ de acrilamida/bisacrilamida. Para la estimación de las masas relativas 
moleculares se utilizaron marcadores de peso molecular (PM) (Sigma) o Fracción F(ab’) 2 de inmunoglobulinas equinas (100 kDa), seroalbúmina bovina (Sigma) (67 kDa), y subunidad $\mathrm{B}$ de crotoxina de Crotalus (C.) durissus terrificus (14 kDa). Para el estudio de la actividad fibrino(geno)lítica las muestras del fibrinógeno digerido se corrieron en condiciones reductoras y no reductoras en geles al $10 \%$. En este caso se utilizó como marcador de PM el kit Pre-Stained Broad Range (Bio Rad). Tras las electroforesis se tiñeron las proteínas con Azul brillante de Coomassie (Bio Rad).

Cromatografía por filtración en gel: se realizó en un sistema FPLC (Pharmacia LKB) utilizando una columna preempacada de 10 x 300 mm de Superosa 6HR (Pharmacia). Muestras de $5 \mathrm{mg}$ del veneno disueltos en $\mathrm{NaCl} 0.2 \mathrm{M}$ y filtrados por $0.2 \mu \mathrm{m}$ se inyectaron en la columna preequilibrada con la misma solución. Las corridas se realizaron a un flujo de $0.4 \mathrm{ml} / \mathrm{min}$, se registró el eluido de la columna a $280 \mathrm{~nm}$ y se separaron los diferentes picos cromatográficos. A los mismos se les determinaron las actividades: hemorrágica, necrotizante, coagulante en plasma, trombina-símil y hemolítica indirecta. Adicionalmente se realizó el SDS-PAGE de las diferentes fracciones obtenidas.

Determinación de las actividades biológicas del veneno crudo: se determinaron las actividades coagulante en plasma (dosis mínima coagulante en plasma: DMC-P), dosis mínima procoagulante en fibrinógeno (DMC-F), dosis mínima desfibrinogenante (DMD), dosis mínima necrotizante (DMN) y dosis mínima hemorrágica (DMH) en ratones CF-1 de acuerdo con las recomendaciones de la Organización Mundial de la Salud (WHO 1981, Theakston y Reid 1983). La actividad hemolítica indirecta de determinó en medio líquido (Al-Abdulha et al. 1991) o por hemólisis radial en medio sólido (Dias Da Silva et al. 1989). La actividad hemolítica directa se determinó de la misma manera que la descrita para la indirecta pero sin agregar fosfolípidos a los medios. La actividad fibrino(geno)lítica se determinó mediante la digestión del fibrinógeno por observación del patrón electroforético de degradación (Sánchez et al. 1995).

Determinación de las actividades biológicas de las fracciones cromatográficas: las actividades hemorrágica y necrotizante se determinaron por la inyección de 50-150 $\mu \mathrm{l}$ de cada fracción por la vía intradérmica (id) en ratas Wistar. Los animales fueron sacrificados a las $3 \mathrm{~h} \mathrm{o}$ a las $72 \mathrm{~h}$ con éter etílico y se midieron en la faz dérmica las lesiones hemorrágicas o necróticas respectivamente. La determinación de las actividades coagulante y trombina-símil se realizó por el tratamiento de $0.3 \mathrm{ml}$ de plasma humano o fibrinógeno bovino con 50 o $100 \mu \mathrm{l}$ de cada fracción y controlando el tiempo de formación del coágulo hasta los $10 \mathrm{~min}$. La presencia de actividad hemolítica indirecta se determinó agregando 5-15 $\mu \mathrm{l}$ de cada fracción a un sistema conteniendo eritrocitos humanos, yema de huevo $5 \% \mathrm{v} / \mathrm{v}$ y $\mathrm{CaCl}_{2} 10 \mathrm{mM}$ en un volumen final de $1 \mathrm{ml}$. Tras una incubación de $2 \mathrm{~h}$, se centrifugaron los tubos y se determinó la actividad peroxidásica del sobrenadante de cada fracción, de acuerdo a métodos descritos en trabajos previos (de Roodt et al. 2000).

Antivenenos: todos los antivenenos utilizados fueron de origen equino.

- $\quad$ Antiveneno Bivalente (BIV): desarrollado por el Instituto Nacional de Producción de Biológicos -A.N.L.I.S. "Dr. Carlos G. Malbrán” (I.N.P.B.), lote 252, fecha de vencimiento 07/2002. Inmunógenos utilizados: venenos de B. alternatus ("yarará grande”, “víbora de la cruz”) y B. neuwiedii ("yarará chica” o "yarará overa”) (de Roodt et al. 1996).

- $\quad$ Antiveneno Tetravalente (TTV): producido por el I.N.P.B, lote 423, fecha de vencimiento 04/2001. Inmunógenos utilizados: veneno de las dos especies mencionadas anteriormente más los venenos de B. jararaca ("yararaca”, "perezosa”) y B. jarara- 
cussu (“yararacuzú”, “tapete dourado”) (de Roodt et al. 1998).

- Suero antiofídico polivalente "Biol" (BIOL):producido por el Instituto Biológico Argentino S.A.I.C., lote 004, fecha de vencimiento 11/2004. Inmunógenos utilizados: venenos de $C$. durissus ("víbora de cascabel”), B. alternatus y B. neuwiedii.

- $\quad$ Suero Antibotrópico (AB): producido por el Instituto Butantan, Sao Paulo, Brasil, lote 9703038, fecha de vencimiento 03/2000. Inmunógenos utilizados: venenos de $B$. jararaca (50\% de la mezcla inmunogénica) y $B$. jararacussu, $B$. neuwiedii, B. alternatus y B. moojeni $(12.5 \%$ de la mezcla total de cada uno) (Dias Da Silva et al. 1989).

- Suero Antiofídico Polivalente (PROB): producido por el Laboratorio Probiol, Bogotá, Colombia, lote APO 441V03, fecha de vencimiento 2003. Inmunógenos utilizados: venenos de $B$. asper, $B$. atrox, L. muta y C. d. terrificus.

- Suero Antiofídico Polivalente (SAP): producido por el Centro de Biotecnología, Facultad de Farmacia, Universidad Central de Venezuela, Venezuela, lote 111, fecha de vencimiento 05/2003. Inmunógenos utilizados: venenos de $B$. colombiensis y C. durissus terrificus.

Para los ensayos inmunoquímicos se utilizaron todos los antivenenos mientras que para los ensayos de neutralización los antivenenos BIV, TTV y BIOL.

Estudio inmunoquímicos: se determinó la reactividad inmunoquímica del veneno de B. cotiara frente a todos los antivenenos mencionados según lo descrito por Theakston et al. (1977) con algunas modificaciones (de Roodt 2002). Se sensibilizaron placas de ELISA de 96 pocillos (Corning) con veneno de $B$. cotiara a una concentración de $1 \mu \mathrm{g} / \mathrm{ml}$. En cada placa se colocaron $100 \mu$ de solución de cada V por pocillo. Las placas así sensibilizadas se dejaron toda la noche a $4{ }^{\circ} \mathrm{C}$ y se lavaron tres veces durante 5 min con TBS $0.05 \mathrm{M} \mathrm{pH}$ 8.0Tween 20 al $0.05 \%$. Posteriormente las placas se incubaron con solución de bloqueo $(100 \mu \mathrm{l}$ por pocillo de TBS pH 8.0 con seroalbúmina bovina al $10 \%$ ) durante toda la noche a $4{ }^{\circ} \mathrm{C}$ a fin de bloquear las uniones inespecíficas, tras lo cual se lavaron con PBS-Tween, con tres lavados de 5 min cada uno. Se realizaron diluciones seriadas de los diferentes antivenenos y de suero normal equino (SNE) como control negativo, y se incubó durante $30 \mathrm{~min}$ a $37^{\circ} \mathrm{C}$, tras lo cual se realizaron tres lavados de 5 min con TBS-Tween-BSA. Tras los lavados se colocó en cada pocillo $100 \mu \mathrm{l}$ de anti-IgGTotal de equino (Sigma) en una dilución de 1/5000 en TBS-Tween-BSA. Se incubó $30 \mathrm{~min}$ a $37^{\circ} \mathrm{C}$ tras lo cual se realizaron cuatro lavados (los dos primeros con TBS-Tween-BSA y los dos últimos con TBS solo). La reacción se reveló con ATBS (Sigma) incubándose $10 \mathrm{~min}$ en la oscuridad a temperatura ambiente y se detuvo con $\mathrm{H}_{2} \mathrm{SO}_{4} 3.0 \mathrm{~N}$ tras lo cual se realizó la lectura a una longitud de onda de $405 \mathrm{~nm}$. El análisis de la absorbancia en función de la dilución de los antivenenos se realizó mediante la utilización del software Prism (GraphPad Inc., CA).

Neutralización de la actividad letal: se inyectaron ratones CF-1 de 18-20 g con $180 \mu \mathrm{g}$ $\left(3.5 \mathrm{DL}_{50}\right.$ ) de veneno diluido en $\mathrm{NaCl} 0.15 \mathrm{M}$ (control positivo) o preincubado por $30 \mathrm{~min}$ a $37^{\circ} \mathrm{C}$ con distintas cantidades de los diferentes antivenenos por la vía ip. Para cada nivel de dosis de antiveneno se utilizaron grupos de 8 ratones. La potencia neutralizante se expresó como Dosis Eficaz $50 \%\left(\mathrm{DE}_{50}\right)$, que indica la cantidad de antiveneno que protege a la mitad de los ratones desafiados, la que se calculó por regresión no lineal a partir de la curva Dosis de Antiveneno versus porcentaje de protección, utilizando el Software Prisma Versión 3.

Neutralización de las actividades hemorrágica y necrotizante: se realizó de acuerdo con la técnica descrita por Ferreira 
y colaboradores con algunas modificaciones (Ferreira et al. 1992, de Roodt et al. 1999). Brevemente, grupos de tres ratones (CF-1, 18$22 \mathrm{~g}$ de peso) fueron inoculados por la vía id con $85 \mu \mathrm{g}$ de veneno (4.2 DMH) o con $100 \mu \mathrm{g}$ de veneno (2.6 DMN) preincubados con diferentes dosis de antivenenos a $37^{\circ} \mathrm{C}$ durante 30 min. Como controles positivos se inocularon ratones con 4.2 DMH o 2.6 DMN en $100 \mu \mathrm{l}$ de $\mathrm{NaCl} 0.15 \mathrm{M}$. A las $3 \mathrm{~h}$ (para actividad hemorrágica) o a las 72 h (para actividad necrotizante) los ratones fueron sacrificados y se determinaron las potencias hemorrágica o necrotizante por la medición de los diámetros perpendiculares mayores de las lesiones de acuerdo con Theakston y Reid 1983. Se asignó un valor de $100 \%$ a la media del área de los controles positivos y las $\mathrm{DE}_{50}$ de cada antiveneno (dosis que reduce la lesión hemorrágica al $50 \%$ ) se determinaron por el análisis de regresión no lineal de la curva de inhibición del área hemorrágica o necrótica en función de las dosis de antiveneno, de manera similar a lo antes mencionado.

Neutralización de la actividad procoagulante: para determinar la neutralización de la actividad coagulante sobre plasma, $150 \mu \mathrm{g}$ de veneno (3.6 DMC-P) se incubaron con diferentes dosis de los antivenenos (100, 50, 20 y $5 \mu \mathrm{l}$ ) durante $30 \mathrm{~min}$ a $37^{\circ} \mathrm{C}$, tras lo cual se agregaron a tubos conteniendo $500 \mu \mathrm{l}$ de plasma humano normal con una concentración de fibrinógeno de $2.8 \mathrm{~g} / \mathrm{dl}$. Para determinar la neutralización de la actividad trombina-símil, $300 \mu \mathrm{g}$ de veneno (2.3 DMC-F) se incubaron con diferentes dosis de los antivenenos (200, 100,50 y $25 \mu \mathrm{l}$ ) durante $30 \mathrm{~min}$ a $37^{\circ} \mathrm{C}$, tras lo cual se agregaron a tubos conteniendo $500 \mu \mathrm{l}$ de una solución de fibrinógeno bovino al $2 \%$ en $\mathrm{NaCl} 0.15 \mathrm{M}$. En ambos casos se determinó el tiempo de coagulación de las diferentes muestras y se consideró como medida de la potencia neutralizante la menor dosis de antiveneno que inhibió la formación del coágulo por al menos $5 \mathrm{~min}$. Como controles negativos se adicionaron $200 \mu$ l de cada antiveneno a tubos conteniendo $500 \mu \mathrm{l}$ de plasma humano o fibrinógeno al 2 \% controlándose la ausencia de coagulación tras los 60 min de la adición, como mínimo.

\section{Neutralización de la actividad hemolíti-} ca indirecta: se preincubaron $25 \mu \mathrm{g}$ de veneno (2.5 dosis hemolíticas indirectas) con diferentes dosis de los antivenenos $(100,50,20,10,5$ y $1 \mu \mathrm{l}$ ) durante $30 \mathrm{~min}$ a $37^{\circ} \mathrm{C}$, tras los cuales se colocó la mezcla preincubada en un sistema constituido por $100 \mu \mathrm{l}$ de glóbulos rojos de carnero lavados, $100 \mu \mathrm{l}$ de yema de huevo y $\mathrm{NaCl} 0.15 \mathrm{M}$ hasta volumen final de $10 \mathrm{ml}$ (sistema hemolítico). El sistema con el veneno-antiveneno preincubado se incubó durante $120 \mathrm{~min}$ a $37^{\circ} \mathrm{C}$. Tras la incubación, se frenó la reacción con EDTA $10 \mathrm{mM}$, se centrifugó a 2000 g durante $10 \mathrm{~min}$ tras lo cual se leyó la absorbancia del sobrenadante a $550 \mathrm{~nm}$. Como controles se utilizaron tubos con el sistema hemolítico tratados con $10 \mu \mathrm{l}$ de $\mathrm{NaCl} 0.15 \mathrm{M}$ (control negativo) o con $10 \mu \mathrm{l}$ de $\mathrm{NaCl} 0.15$ $\mathrm{M}$ conteniendo $25 \mu \mathrm{g}$ de veneno de $B$. cotiara (control positivo). La capacidad neutralizante se expresó como $\mathrm{DE}_{50}$, considerándose ésta como la dosis de antiveneno que reduce la absorbancia respecto a los controles positivos en un 50 \%. Ésta fue determinada por el análisis por regresión no lineal de la curva dosis versus inhibición de la hemólisis de acuerdo con lo descrito anteriormente.

\section{RESULTADOS}

El patrón cromatográfico del veneno puso en evidencia seis picos, de los cuales dos (correspondientes a las fracciones I y II) están en mayor proporción y eluyen al inicio de la corrida y dos picos importantes (correspondientes a las fracciones III y IV) que se observan antes de su finalización (Fig. 1). Las actividades hemorrágica y necrotizante se observaron en el pico inicial, la actividad procoagulante se observó hasta el segundo pico y la hemolítica indirecta en los picos tercero y cuarto, características similares a las también observadas en otros venenos de Bothrops de la Argentina (de Roodt et al. 1996, 1998, de 


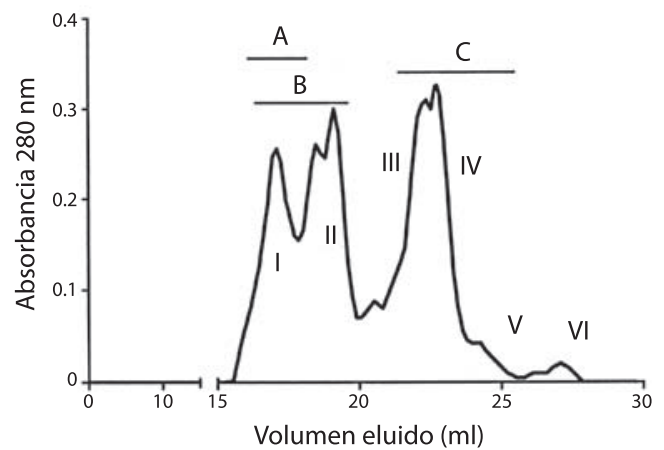

Fig. 1. Fracciones cromatográficas del veneno de $B$. cotiara. Perfil cromatográfico del veneno de $B$. cotiara corrido en una columna de Superosa 6 HR $(10$ x 300 mm) equilibrada con $\mathrm{NaCl} 0.2 \mathrm{M}$ y a un flujo de $0.4 \mathrm{ml} / \mathrm{min}$. Los números romanos indican las fracciones en las que se realizaron los ensayos de actividades biológicas. Las letras indican las actividades encontradas según el volumen de elución: A. Actividades hemorrágica y necrotizante en piel. B. Actividad coagulante en plasma y trombina símil. C. Actividad hemolítica indirecta.

Fig. 1. Chromatographic fractions of B. cotiara venom. Chromatographic profile of $B$. cotiara venom in a Superose $6 \mathrm{HR}(10 \times 300 \mathrm{~mm})$ equilibrated with $0.2 \mathrm{M} \mathrm{NaCl} 0.2 \mathrm{M}$ with a flow rate of $0.4 \mathrm{ml} / \mathrm{min}$. Roman numbers indicate the fractions on which the assays of biological activities were done. Letters indicate the activities found regarding the elution volume. A. Hemorrhagic and necrotizing activity on skin. B. Coagulant and thrombin like activities. C. Indirect hemolytic activity.

Roodt 2002). El SDS-PAGE de las fracciones del veneno puso en evidencia bandas de PM cercano a los $60 \mathrm{kDa}$ en las fracciones con actividad hemorrágica y necrotizante, bandas entre 24 y 50 kDa con relación a las actividades coagulantes y trombina-símil y bandas entre los 24 y 14 kDa con relación a la actividad hemolítica indirecta (Fig. 2).

Las actividades biológicas determinadas para este veneno fueron $\mathrm{DL}_{50}=51 \mu \mathrm{g}$ (ic $95 \%$ 46-56), $\mathrm{DMH}=20 \pm 2 \mu \mathrm{g}, \mathrm{DMC}-\mathrm{P}=2.1$ $\mathrm{mg} / \mathrm{l}(41 \pm 10 \mu \mathrm{g}$ en $200 \mu \mathrm{l}), \mathrm{DMC}-\mathrm{F}=6.4 \mathrm{mg} / \mathrm{l}$ $(128 \pm 7 \mu \mathrm{g}$ en $200 \mu \mathrm{l}), \mathrm{DMD}=1.0 \pm 0.5 \mu \mathrm{g}$, $\mathrm{DMN}=38 \pm 3 \mu \mathrm{g}$, hemólisis indirecta en medio líquido $=10 \mu \mathrm{g}$ (8-12), hemólisis indirecta radial $=10 \pm 2 \mu \mathrm{g}$. No se detectó actividad hemolítica directa.

Se observó digestión del fibrinógeno. El patrón electroforético de los controles negativos

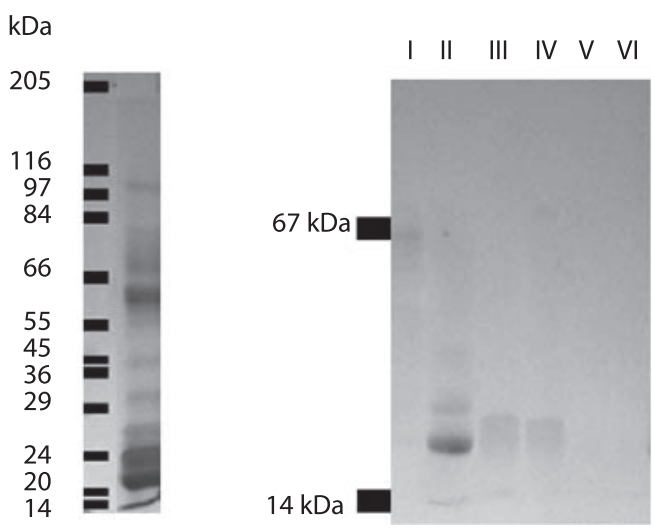

Fig. 2. SDS-PAGE del veneno de $B$. cotiara y sus fracciones cromatográficas. A. SDS-PAGE en condiciones no reductoras del veneno entero de $B$. cotiara. En un gel con una concentración de $12.5 \%$ de acrilamida / bisacrilamida se corrieron $20 \mu \mathrm{g}$ de veneno crudo de B. cotiara; a la izquierda se indican los marcadores de peso molecular (Sigma, Wide Range). B. SDS-PAGE de las fracciones cromatográficas del veneno de B. cotiara, corrido en una columna de Superosa 6 HR. Los marcadores de peso molecular se indican a la izquierda y son correspondientes a la subunidad B de crotoxina (14 kDa) y a seroalbúmina bovina (67 kDa). Los números romanos son correspondientes a las fracciones cromatográficas que se observan el la Fig. 1.

Fig. 2. SDS-PAGE of $B$. cotiara venom and its chromatographic fractions. A. SDS-PAGE in non reducing conditions of the whole venom of $B$. cotiara. Twenty micrograms of crude venom of $B$. cotiara were run in a $12.5 \%$ of acrylamide / bisacrylamide gel in non reducing conditions. The molecular weight markers (Sigma, Wide Range) are indicated in the left. B. SDS-PAGE of the chromatographic fractions of $B$. cotiara venom, analyzed in a Superose $6 \mathrm{HR}$ column. Molecular weight markers are indicated in the left and correspond to the B subunit of crotoxin (14 kDa) and bovine seroalbumin (67 kDa). Roman numbers correspond to the chromatographic fractions observed in Fig. 1.

presentó tres bandas mayores entre los 60 y $35 \mathrm{kDa}$, en correspondencia a las subunidades $\alpha, \beta$ y $\gamma$ del fibrinógeno (Santoro y Sano Martins 1993). Las muestras incubadas con veneno, presentaron bandas más fuertemente teñidas por debajo de los $35 \mathrm{kDa}$. En las muestras tratadas con veneno, no se observaron las bandas en correpondencia a la subunidad $\alpha$ del fibrinógeno. En estas muestras también se pudo apreciar la hidrólisis parcial de las bandas correspondientes a la subunidad $\beta$ del fibrinógeno (Fig. 3). 


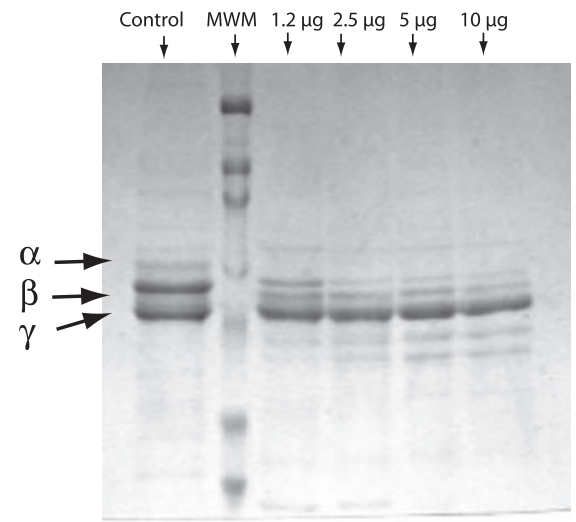

$\mathrm{kDa}$

205

124

80

49.1

34.8

28.9

20.6

9.4

Fig. 3. Hidrólisis del fibrinógeno bovino por el veneno de $B$. cotiara. SDS-PAGE en condiciones reductoras de la digestión de fibrinógeno por veneno de $B$. cotiara. A tubos con $2 \mathrm{ml}$ de solución de fibrinógeno bovino al 2\% (Sigma) en $\mathrm{NaCl} 0.15 \mathrm{M}$ se le agregaron 1.2, 2.5, 5 ó $10 \mu \mathrm{g}$ de veneno (cantidades expresadas en las calles correspondientes en el gel). Se incubaron a $37^{\circ} \mathrm{C}$ durante $3 \mathrm{~h}$, tras lo cual se tomaron alícuotas que se estudiaron en condiciones reductoras por SDS-PAGE (geles al 10\%). Como control se utilizó fibrinógeno bovino tratado en las mismas condiciones sin el agregado de veneno (primer calle a la izquierda), indicando las bandas $\alpha, \beta$ y $\gamma$. En la segunda calle a la izquierda se sembró el estándar de peso molecular (Bio Rad Pre Stained, Broad Range). Los valores de masa molecular de las proteínas estándar se indican a la derecha.

Fig. 3. Hidrolytic activity on bovine fibrinogen. SDSPAGE in reducing conditions of bovine fibrinogen incubated with $B$. cotiara venom. Tubes containing $2 \mathrm{ml}$ of $2 \%$ bovine fibrinogen (Sigma) in $\mathrm{NaCl} 0.15 \mathrm{M}$, were treated with 1.2, 2.5, 5 or $10 \mu \mathrm{g}$ of venom (the amount is expressed in the lanes). Samples were incubated $3 \mathrm{~h}$ at $37^{\circ} \mathrm{C}$ after which, aliquots were studied in reducing conditions by SDS-PAGE (10\% Acrilamyde/bis-acriylamide). As control was used bovine fibrinogen in the same conditions without the addition of venom (first lane at the left) indicating the bands of $\alpha, \beta$ and $\gamma$ fibrinogen chains. In the second lane are indicated the molecular weight markers (Bio Rad, Pre Stained, Broad Range), the mass of the molecular weight markers are indicated in the right.

Todos los antivenenos mostraron una reactividad inmunoquímica alta frente a este veneno. En la dilución de 1 x $10^{-2}$ presentaron el máximo de absorbancia, sin embargo la dilución de antiveneno que corresponde al $50 \%$ de la absorbancia máxima fue distinta para todos los antivenenos, siendo las más potentes las del BIV (1132 [ic $95 \%$ 630-2033]) y el

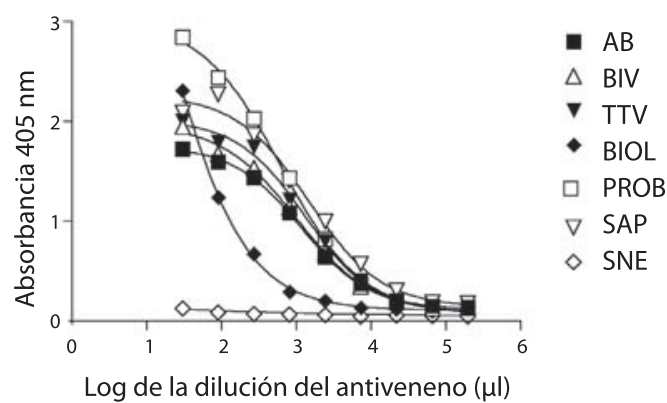

Fig. 4. ELISA de antivenenos frente al veneno de B. cotiara. Se evaluaron varios antivenenos para uso terapéutico en su reactividad inmunoquímica frente al veneno de $B$. cotiara. Se indica la absorbancia a $405 \mathrm{~nm}$ del logaritmo de las diluciones de cada antiveneno. AB: Antibotrópico del Instituto Butantan, Brasil; BIV: Bivalente del INPB-ANLIS "Dr. Carlos G. Malbrán”, Argentina; TTV: Tetravalente del INPB-ANLIS. "Dr. Carlos G. Malbrán”, Argentina; BIOL: Antiveneno polivalente Biol, Argentina; PROBIOL: antiveneno polivalente de Probiol, Colombia; SAP: Suero antiofídico polivalente del Centro de Biotecnología, Universidad Central, Venezuela. SNE: suero normal equino.

Fig. 4. ELISA of the different antivenoms against $B$. cotiara venom. The immunochemical reactivity of several therapeutic antivenoms against $B$. cotiara venom was studied. Figure shows the absorbance at $405 \mathrm{~nm}$ of the $\log$ of the dilution of each antivenom. AB: Antibotrópico from Butantan Institute, Brazil; BIV: Bivalente from INPB-ANLIS “Dr. Carlos G. Malbrán”, Argentina; TTV: Tetravalente from INPB-ANLIS “Dr. Carlos G. Malbrán”, Argentina; BIOL: Antiveneno Polivalente Biol, Argentina; PROBIOL: polyvalent antivenom of Probiol, Colombia; SAP: Suero Antiofídico Polivalente from the Centro de Biotecnología, Universidad Central, Venezuela. SNE: normal equine serum.

TTV (1126[853-1487]), seguidas por las del AB (800 [450-1421]), mientras que el BIOL fue el que presentó menor reactividad (74 [49112]) (Fig. 4).

Los resultados de los ensayos de neutralización son presentados en el Cuadro 1 . Se observaron diferencias en la potencia neutralizante de las distintas actividades, si bien en la mayoría de los casos estas diferencias no fueron significativas. La potencia letal de los venenos fue neutralizada por los tres antivenenos. El TTV mostró una $\mathrm{DE}_{50}$ algo más potente (30 \%) que el BIV y el BIOL, aunque las diferencias no poseen significancia estadística (Cuadro 1). Las actividades hemorrágica y necrotizante 
CUADRO 1

Resultado de los ensayos de neutralización del veneno de Bothrops cotiara utilizando tres antivenenos diferentes

TABLE 1

Results from the assays of neutralization capacity for three antivenoms on Bothrops cotiara venom

\begin{tabular}{|c|c|c|c|c|c|c|}
\hline Suero $^{1}$ & $\begin{array}{l}\text { Letalidad } \\
\qquad(\mu \mathrm{l})\end{array}$ & $\begin{array}{c}\text { Hemorragia } \\
(\mu \mathrm{l})\end{array}$ & $\begin{array}{l}\text { Necrosis } \\
(\mu \mathrm{l})\end{array}$ & $\begin{array}{c}\text { Actividad } \\
\text { Trombina-símil } \\
(\mu \mathrm{l})\end{array}$ & $\begin{array}{l}\text { Actividad } \\
\text { Coagulante en } \\
\text { Plasma } \\
(\mu \mathrm{l})\end{array}$ & $\begin{array}{l}\text { Hemólisis Indirecta } \\
\qquad(\mu \mathrm{l})\end{array}$ \\
\hline BIV & $\begin{array}{c}10.29 \\
(9.8-10.7)\end{array}$ & $\begin{array}{c}1.29 \\
(1.0-1.6)\end{array}$ & $\begin{array}{c}1.14 \\
(0.4-2.7)\end{array}$ & 100 & 20 & $\begin{array}{c}4.6 \\
(4.5-4.7)\end{array}$ \\
\hline TTV & $\begin{array}{c}7.59 \\
(5.6-10.1)\end{array}$ & $\begin{array}{c}1.75 \\
(1.6-1.9)\end{array}$ & $\begin{array}{c}1.71 \\
(0.7-3.7)\end{array}$ & 100 & 20 & $\begin{array}{c}4.6 \\
(4.5-4.7)\end{array}$ \\
\hline BIOL & $\begin{array}{c}11.39 \\
(9.7-13.4)\end{array}$ & $\begin{array}{c}2.27 \\
(1.5-3.4)\end{array}$ & $\begin{array}{c}2.36 \\
(1.3-4.0)\end{array}$ & $>200$ & 50 & $\begin{array}{c}20.2 \\
(16.1-25.2)\end{array}$ \\
\hline
\end{tabular}

${ }^{1}$ La potencia neutralizante de los antivenenos indica la capacidad neutralizante de cada antiveneno sobre letalidad, hemorragia, necrosis en piel y potencia hemolítica indirecta del veneno. La potencia neutralizante está indicada como dosis efectiva media $\left(\mathrm{DE}_{50}\right)$ en $\mu \mathrm{l}$ de antiveneno y se expresa como la media y el intervalo de confianza de 95\% entre paréntesis. En la neutralización de las actividades coagulante y trombina símil, la dosis corresponde a la cantidad de antiveneno que inhibió la formación de coágulo por al menos $5 \mathrm{~min}$.

\begin{abstract}
${ }^{1}$ The neutralizing potency of the antivenoms indicates the neutralizing capacity of each antivenom on the lethality, hemorrhage, necotizing activity on skin and the indirect hemolytic activity of the venom from $B$. cotiara. The neutralizing potency is indicated as the median effective dose $\left(\mathrm{ED}_{50}\right)$ expressed in $\mu \mathrm{l}$ of antivenom as the mean and the $95 \%$ confidence intervals (into brackets). The neutralization of the coagulant and thrombin like activity is indicated as the minimal dose of antivenom that inhibits the clot formation at least by 5 min and is expressed in $\mu \mathrm{l}$.
\end{abstract}

fueron neutralizadas bien por todos los antivenenos, si bien el BIV en orden de $\mathrm{DE}_{50}$ tuvo una potencia superior al $25 \%$ respecto al TTV y de más del $50 \%$ respecto al BIOL (Cuadro 1). Los antivenenos BIV y TTV neutralizaron la actividad coagulante sobre plasma y sobre fibrinógeno en forma similar, mientras que el BIOL no fue efectivo con las dosis utilizadas para neutralizar la actividad trombina-símil y requirió más del doble de dosis para neutralizar la actividad procoagulante en plasma (Cuadro 1). Los antivenenos BIV y TTV fueron mas efectivos que el BIOL en la neutralización de la actividad hemolítica con una $\mathrm{DE}_{50}$ cuatro veces más potente $(\mathrm{p}<0.05)$ (Cuadro 1$)$.

\section{DISCUSIÓN}

Los perfiles cromatográfico y electroforético, así como las actividades biológicas de las diferentes fracciones cromatográficas, mostraron en líneas generales similitud a los descritos para los venenos de otras especies de Bothrops de la Argentina (de Roodt et al. 1996, 2000, de Roodt 2002).

Las principales actividades tóxicas tuvieron potencias dentro de los rangos descritos para los venenos de Bothrops. Por ejemplo, la $\mathrm{DL}_{50}$ ip estuvo dentro de los valores de aquellas comunicadas para los venenos de las otras especies de este género en la Argentina, que oscilan entre 18 y $73 \mu$ g (de Roodt 2002) y para las de los venenos de otras especies de Bothrops de Sudamérica que oscilan entre 38 y $230 \mu \mathrm{g}$ (Sánchez et al. 1992), si bien su potencia desfibrinogenante parecería ser de las más altas dentro de las halladas para estas serpientes (Sanchez et al. 1992, de Roodt 2002).

Dado que las mordeduras por Bothrops representan la mayoría de los casos de envenenamientos por mordeduras de serpientes venenosas en Centro y Sudamérica, los antivenenos botrópicos son los más utilizados a nivel 
asistencial en estas regiones. Sin embargo, ninguno de los antivenenos disponibles en Sudamérica se produce utilizando en la mezcla inmunogénica el veneno de $B$. cotiara. Si bien la alta neutralización cruzada que confieren los antivenenos botrópicos es un hecho ampliamente conocido (Siles Villarroel et al. 1978/79, Dias Da Silva et al. 1989, de Roodt et al. 1997, 1998, 1999, Bogarín et al. 2000), la capacidad neutralizante de las actividades tóxicas de los diferentes venenos por los antivenenos a ser utilizados terapéuticamente debe ser demostrada experimentalmente según las recomendaciones de la OMS (WHO 1981, Theakston y Reid 1983).

Todos los antivenenos estudiados mostraron una gran reactividad inmunoquímica frente a este veneno, independientemente de las mezclas inmunogénicas utilizadas para su producción y presentación farmacéutica. La potencia letal y las diferentes actividades tóxicas fueron bien neutralizadas por los tres antivenenos estudiados, sin encontrarse diferencias importantes, con excepción de las observadas en las actividades coagulantes y fosfolipásica indirecta, ante las cuales el antiveneno BIOL fue menos efectivo. Si bien todos los antivenenos neutralizaron la actividad coagulante, los antibotrópicos se mostraron más potentes. El antiveneno TTV, desarrollado con el veneno de cuatro de las serpientes de Misiones, presentó una potencia neutralizante de la letalidad mayor y el BIV fue más efectivo en neutralizar las actividades hemorrágica y necrótica del veneno, si bien las dosis no fueron diferentes estadísticamente (Cuadro 1).

La hemorragia es una de las actividades tóxicas más importantes de los venenos botrópicos (Ferreira et al. 1992, de Roodt et al. 2003) y una de las manifestaciones clínicas más frecuentes en estos envenenamientos (Silva Haad 1980/81, Otero et al. 1997, Ribeiro y Jorge 1997, Rodríguez Acosta et al. 2000). Los antivenenos se mostraron efectivos en la neutralización de la actividad hemorrágica, lo que indica la neutralización de las metaloproteinasas (hemorraginas o reprolisinas) del veneno. Esto era de esperar dada la alta similitud entre las diferentes metaloproteinasas (Bjarnasson y Fox 1994) y al hecho de que para este veneno se han descrito componentes hemorrágicos (Pessatti et al. 1995) que presentan relación antigénica con la bothrojaragina de $B$. jararaca (Castro et al. 1999).

Otra característica destacada de los venenos botrópicos es su capacidad para producir necrosis (Silva Haad 1980/81, Gutiérrez y Lomonte 1990, Ministerio de Saude 1999, Gutiérrez y Lomonte 2003) por acción conjunta de varios de sus componentes (Mebs y Ownby 1990, Gutiérrez y Lomonte 1990, 2003). Las fosfolipasas de las Bothrops son citotóxicas y producen lesiones en células musculares y otros elementos celulares (Gutiérrez y Lomonte 1995, Fletcher y Jiang 1998), mientras que las metaloproteinasas destruyen la matriz extracelular y vasos sanguíneos (Bjarnasson y Fox 1994, Gutiérrez y Rucavado 2000). Por otro lado, la formación de trombos y émbolos producidos por los componentes que actúan sobre el sistema hemostático (Markland 1998) provocan alteraciones en la microcirculación que conducen conjuntamente con el daño a los vasos sanguíneos y la destrucción de la matriz extracelular, a procesos isquémicos responsables de fenómenos necróticos (Queiroz y Petta 1984), por lo que la necrosis tisular puede producirse tanto por acción directa como indirecta de los diferentes componentes de los venenos botrópicos (Gutiérrez y Lomonte 2003). Los antivenenos estudiados, mostraron ser capaces de neutralizar los componentes del veneno responsables de los procesos hemorrágicos, coagulantes, necróticos en piel y al menos a las fosfolipasas D49, con actividad enzimática (fosfolipasas $\mathrm{A}_{2}$ aspartato-49). Las fosfolipasas responsables de los fenómenos necróticos provocados por los venenos de las Bothrops no necesariamente poseen alta actividad enzimática. Como ejemplos de esto se puede mencionar el caso del veneno de $B$. alternatus que posee una fosfolipasa acídica con una actividad muy baja (Nisembon et al. 1986) o el de las fosfolipasas K49 (fosfolipasas $A_{2}$ lisina-49) que se encuentran en gran cantidad en muchas especies de Bothrops, que no poseen actividad 
fosfolipásica (Gutiérrez y Lomonte 1995). Por este motivo, no necesariamente la actividad fosfolipásica tiene que relacionarse con la capacidad necrotizante de un veneno, aunque pueda tener intervención en esta.

Los antivenenos botrópicos fueron más eficientes que el BIOL en neutralizar la actividad hemolítica indirecta. Una de las posibles explicaciones de esto podría ser que los antivenenos botrópicos poseen mayor cantidad de anticuerpos contra fosfolipasas de venenos de Bothrops. El antiveneno botrópico-crotálico, en su mezcla inmunogénica posee no solo veneno de Bothrops sino también de C. d. terrificus, cuya fosfolipasa (crotoxina) aunque cruza inmunoquímicamente con las fosfolipasas de las Bothrops (de Roodt et al. 1998) es muy diferente a la mayoría de estas. Por otro lado, se describió una mejor neutralización de la actividad fosfolipásica de los venenos botrópicos por los antivenenos específicos (Ferreira et al. 1992).

La información obtenida de los ensayos de neutralización es importante a nivel sanitario, dada la similitud en las formulaciones de los antivenenos disponibles en esa región. Independientemente de las proporciones de cada veneno, las mezclas inmunogénicas con las que se producen estos antivenenos son bastante similares, lo que indicaría que los mismos podrían ser útiles ante la mordedura de $B$. cotiara. Refuerza esto el hecho de que las potencias neutralizantes del BIV, TTV y el AB, son bastante similares, independientemente de las diferencias en las proporciones de los venenos de sus mezclas inmunogénicas (de Roodt 2002).

Esta es la primera comunicación acerca de las actividades tóxicas del veneno de $B$. cotiara de la Argentina y de la capacidad neutralizante de los antivenenos utilizados para el tratamiento de los accidentes botrópicos en la región dónde se encuentra esta serpiente. Las evidencias experimentales indican la utilidad de los antivenenos botrópicos o botrópico-crotálicos sobre las actividades tóxicas del veneno de esta serpiente, de hallazgo mantenido aunque con baja frecuencia de aparición a lo largo de los años.

\section{RESUMEN}

Bothrops cotiara es una serpiente que se encuentra en la provincia de Misiones (Argentina), el Sur de Brasil y Paraguay. La información sobre las características clínicas de los accidentes por esta serpiente es muy escasa y existen pocos datos sobre su veneno y la capacidad neutralizante de las actividades tóxicas del mismo por antivenenos terapéuticos. En este trabajo se estudiaron características bioquímicas, actividades tóxicas y la reactividad inmunoquímica del veneno de B. cotiara. Seis antivenenos anti Viperinos Sudamericanos fueron estudiados frente a este veneno por el método ELISA y se probó la capacidad neutralizante de tres de estos frente a las actividades hemorrágica, necrotizante, procoagulante, trombina-símil, hemolítica indirecta y la potencia letal de veneno de ejemplares de $B$. cotiara de la provincia de Misiones. Los patrones cromatográficos y electroforéticos mostraron características similares a los de otros venenos de Bothrops. Las actividades tóxicas estuvieron dentro de los ámbitos descritos para los venenos botrópicos. Los seis antivenenos mostraron gran reactividad inmunoquímica por ELISA y las potencias neutralizantes de los tres estudiados fueron muy próximas para las actividades letal, hemorrágica, necrotizante, hemolítica indirecta, coagulante y trombina-símil. Los resultados de los estudios de neutralización indicarían que ante la mordedura de esta poco común especie de Bothrops, pueden usarse los diferentes tipos de antivenenos botrópicos o botrópico-crotálicos para uso terapéutico disponibles en esa región.

Palabras clave: víboras, Bothrops cotiara, toxicidad, antiveneno, neutralización.

\section{REFERENCIAS}

Al-Abdulla, I.H., A.M. Sidki \& J. Candon. 1991. An Indirect hemolytic assay for assessing antivenoms. Toxicon 29: 1043-1046.

Alves Araujo, F.A., M. Santalúcia \& R. Fernández Cabral. 2003. Epidemiología dos accidentes por animales peconhentos, p. 6-12. In J.L. Costa Cardoso, F.O. de Siqueira Franca, F.H. Wen, C.M. Sant`Ana Málaque \& V. Haadad Jr. (eds.). Animais Peconhentos no Brasil. Biologia, Clínica e Terapéutica dos Acidentes. Sarvier, FAPESP, Sao Paulo, Brasil.

Bjarnasson, J.B. \& J.W. Fox. 1994. Hemorrhagic metalloproteinases from snake venoms. Pharmac. Ther. 62: 325-372.

Bogarín, G., J.F. Morais, I.K. Yamaguchi, M.A. Stephano, J.R. Marcelino, A.K. Nishikawa, R. Guidolín, 
G. Rojas, H.G. Higashi \& J.M. Gutiérrez. 2000. Neutralization of crotaline snake venoms from Central and South America by antivenoms produced in Brazil and Costa Rica. Toxicon 38: 1429-1441.

Bonnet, E.F.P. \& E.A. Pedace. 1971. Muerte por picadura de víbora yarará. Consideraciones histopatológicas y médico-legales. Bol. Acad. Nac. Med. 48: 417-432.

Castro, H.C., M. Fernandez \& R.B. Zingali. 1999. Identification of bothrojaracin-like proteins in snake venoms from Bothrops species and Lachesis muta. Toxicon 37: 1403-1416.

Costa Cardoso, J.L. \& H.W. Fan 1995. Snakebites in South America, p. 261-329. In J. Meier \& J. White (eds.). Handbook of Clinical Toxicology of Animal Venoms and Poisons. CRC, Boca Raton, Florida, EEUU.

de Roodt, A.R. 2002. Estudio Inmunobiológico del Veneno de las Serpientes de Importancia Sanitaria de la Argentina. Tesis de doctorado, Universidad de Buenos Aires, Buenos Aires. 313 p.

de Roodt, A.R. \& J.C. Troiano. 1995. Identificación de las Serpientes Venenosas de la República Argentina. Clín. Prod. Vet. 24: 16-28.

de Roodt, A.R., J.A. Dolab, S.E. Hajos, T. Fernández \& L. Segre. 1996. Una modalidad diferente de inmunización para la obtención de suero antiofídico botrópico-crotálico en equinos. Rev. Farmac. 138: 9-20.

de Roodt, A.R., J.A. Dolab, S.E. Hajos, T. Fernández \& L. Segre. 1997. Utilidad de los sueros terapéuticos de uso corriente en Argentina frente al envenenamiento por la serpiente Bothrops moojeni (“caisaca”) (Viperidae, Crotalinae). Medicina 57: 667-676.

de Roodt, A.R., J.A. Dolab, T. Fernández, L. Segre \& S.E. Hajos. 1998. Cross reactivity and heterologous neutralization of crotaline antivenoms used in Argentina. Toxicon 36: 1025-1038.

de Roodt, A.R., J.C. Vidal, S. Litwin, J.A. Dolab, S.E. Hajos \& L. Segre. 1999. Neutralización cruzada del veneno de Bothrops jararacussu por sueros antiofídicos heterólogos. Medicina 59: 238-242.

de Roodt, A.R., J.A. Dolab, J.C. Dokmetjian, S. Litwin, L. Segre \& J.C. Vidal. 2000. A comparison of different methods to asses the hemorrhagic activity of Bothrops venoms. Toxicon 38: 865-874.

de Roodt, A.R., S. Litwin \& J.C. Vidal. 2003. Hemorrhagic activity of Bothrops venoms determined by two different methods and relationship with proteolytic activity on gelatin and lethality. Toxicon 41: 949-958.

de Roodt, A.R., J.A. Dolab, E.G. Gould, J.C. Carfagnini, J.C. Dokmetjian, J. Gould, J.C. Troiano, M.
Amoroso, T. Fernández, L. Segre, S.E. Hajos \& J.C. Vidal. 2000. Some Toxic and enzymatic activities of Bothrops ammodytoides (“yarará ñata”) venom. Toxicon 38: 49-62.

Dias Da Silva, W., R. Guidolin, I. Raw, H.G. Higashi, C.P. Caricatti, J.F. Morais, M.L. Lima, I.K. Yamaguchi, A.K. Nishikawa, M.N. Stephano, J.R. Marcelino, J.R. Pinto \& M.J. Santos. 1989. Crossreactivity of horse monovalent antivenoms to venoms of ten Bothrops species. Mem. Inst. Butantan 51: 153-168.

Ferreira, M.L., A.M. Moura Da Silva, F.O.S. Franca, J.L. Cardoso \& I. Mota. 1992. Toxic activities of venoms from nine Bothrops species and their correlation with lethality and necrosis. Toxicon 30: 1063-1068.

Ferreira, M.L., A.M. Moura Da Silva \& I. Mota. 1992. Neutralization of different activities of venoms from nine species of Bothrops snakes by Bothrops jararaca antivenom. Toxicon 30: 1591-1602.

Fletcher, J.E. \& M.S. Jiang. 1998. Lys49 phospholipase $\mathrm{A}_{2}$ lyse cell cultures by two distinct mechanisms. Toxicon 36: 1549-1556.

Francischetti, I.M., H.C. Castro, R.B. Zingali, C.R. Carlini \& J.A. Guimaraes. 1998. Bothrops sp. snake venoms: comparison of some biochemical and physicochemical properties and interference in platelet functions. Comp. Biochem. Physiol. C. Pharmacol. Toxicol. Endocrinol. 119: 21-29.

Freiberg, M.A. 1968. Ofidios ponzoñosos de la Argentina. Ciencia y Técnica 24: 338-353.

Furtado, M.F., M. Maruyama, A.S. Kamiguti \& L.C. Antonio. 1991. Comparative study of nine Bothrops snake venoms from adult female snakes and their offspring. Toxicon 29: 219-226.

García, S.I. 2003. VI Reunión de Expertos en Envenenamiento por animales ponzoñosos. Instituto de Biotecnología de la Universidad Autónoma de México, Cuernavaca, México, 110 p.

Giraudo, A.R. 2001. Serpientes de la selva Paranaense y del Chaco Húmedo. LOLA, Buenos Aires. 285 p.

Gutiérrez, J.M \& B. Lomonte. 1990. Local tissue damage induced by Bothrops snake venoms. A Review. Mem. Inst. Butantan 51: 211-223.

Gutiérrez, J.M. \& B. Lomonte. 1995. Phospholipase $A_{2}$ myotoxins from Bothrops snake venoms. Toxicon 33: $1405-1424$

Gutiérrez, J.M. \& B. Lomonte. 2003. Efectos Locales en el Envenenamiento Ofídico en América Latina, p. 310-323. In J.L. Costa Cardoso, F.O. de Siqueira 
Franca, F.H. Wen, C.M. Sant`Ana Málaque \& V. Haadad Jr. (eds.). Animais Peconhentos no Brasil. Biologia, Clínica e Terapéutica dos Acidentes. Sarvier, FAPESP, Sao Paulo, Brasil.

Gutiérrez, J.M. \& A. Rucavado. 2000. Snake venom metalloproteinases: Their role in the pathogenesis of local tissue damage. Biochimie 82: 841-850.

Instituto Nacional de Producción de Biológicos, Administración Nacional de Laboratorios e Institutos de Salud “Dr. Carlos G. Malbrán”. 1998. Programa Nacional de Ofidismo y Aracneísmo. Vigilancia Epidemiológica y Control de Ofidismo y Aracneísmo. Aspectos Programáticos. Ministerio de Salud y Acción Social, Buenos Aires. 23 p.

Laemmli, U.K. 1970. Cleavage of structural proteins during the assembly of the head bacteriophage T4. Nature 227: 680-685.

Martino, O., H. Mathet, R.D. Masini, A. Ibarra Grasso, R. Thompson, C. Gondell \& J. Bosch. 1979. Emponzoñamiento humano provocado por venenos de origen animal. Secretaría de Salud de la República Argentina, Buenos Aires. 240 p.

Markland, F.S. 1998. Snake venoms and the hemostatic system. Toxicon 36: 1749-1800.

Mebs, D. \& C.L. Ownby. 1990. Myotoxic components of snake venoms: their biochemical and biological activities. Pharmaco. Ther. 48: 223-236.

Ministerio de Bienestar Social, Secretaría de Estado de Salud Pública. 1980. Guía de prevención y tratamiento de las mordeduras por serpientes venenosas. Ministerio de Bienestar Social, Buenos Aires. 47 p.

Ministerio de Salud y Acción Social. 1999. Guía de Centros antiponzoñosos de la República Argentina. Encuesta 1998-1999. Ministerio de Salud de la Nación, Buenos Aires. 102 p.

Ministerio de Saúde. Fundacáo Nacional de Saúde. 1999. Manual de Diagnóstico e Tratamento de Acidentes por Animais Peçonhentos. Ministerio da Saúde. Fundaçáo Nacional de Saúde, Brasilia. 131 p.

Moura da Silva, A.M., D.F. Cardoso \& M.M. Tanizaki. 1990. Differences in distribution of myotoxic proteins in venoms from different Bothrops species. Toxicon 28: 1293-1301.

Moura da Silva, A.M., H. Desmond, G. Laing \& R.D. Theakston. 1991. Isolation and comparison of myotoxins isolated from venoms of different species of Bothrops snakes. Toxicon 29: 713-723.

Nahas, L., A.S. Kamiguti \& M.A. Barros. 1979. Thrombinlike and factor X-activator components of Bothrops snake venoms. Thromb. Haemost. 41: 314-328.
Nisenbom, H.E., C. Seki \& J.C. Vidal. 1986. Phospholipase $\mathrm{A}_{2}$ from Bothrops alternatus (Víbora de la Cruz). Venom purification and some charateristic properties. Toxicon 24: 259-272.

Otero, R., J. Gutierrez, M. Beatriz Mesa, E. Duque, O. Rodriguez, J.L. Arango, F. Gomez, A. Toro, F. Cano, L. Maria Rodriguez, E. Caro, J. Martinez, W. Cornejo, L.M. Gomez, F.L. Uribe, S. Cardenas, V. Nunez \& A. Diaz. 2002. Complications of Bothrops, Porthidium, and Bothriechis snakebites in Colombia. A clinical and epidemiological study of 39 cases attended in a university hospital. Toxicon 40: 1107-1114.

Pessatti, M., J.D. Fontana, M.F. Furtado, M.F. Guimaraes, L.R. Zanette, W.T. Costa \& M. Baron. 1995. Screening of Bothrops snake venoms for L-amino acid oxidase activity. Appl. Biochem. Biotechnol. 51: 197-210.

Queiroz, L.S. \& C.A. Petta. 1984. Histopatological changes caused by venom of urutu snake (Bothrops alternatus) in mouse skeletal muscle. Rev. Inst. Med. Trop. Sao Paulo 26: 247-253.

Ribeiro, L.A. \& M.T. Jorge. 1997. Bites by snakes in the genus Bothrops: a series of 3139 cases. Rev. Soc. Bras. Med. Trop. 30: 475-480.

Rodriguez Acosta, A., W. Uzcategui, R. Azuaje, I. Aguilar \& M.E. Giron. 2000. Análisis clínico y epidemiológico de los accidentes por mordeduras de serpientes del género Bothrops en Venezuela. Rev. Cubana Med. Trop. 52: 90-94.

Rojas, G., G. Bogarin \& J.M. Gutierrez. 1997. Snakebite mortality in Costa Rica. Toxicon 35: 1639-1643.

Russell, F.E., F.G. Walter, T.A. Bey \& M.C. Fernández. 1997. Snakes and snakebite in Central America. Toxicon 35: 1469-1522.

Sanchez, E.F., T.B. Freitas, D.L. Ferreira-Alves, D.T. Velarde, M.R. Diniz, M.N. Cordeiro, G. AgostiniCotta \& C.R. Diniz. 1992. Biological activities of venoms from South American snakes. Toxicon 30: 95-103.

Sanchez, E.F., M.I.E. Costa, C. Chavez Olortegui, M.T. Assakura, F.R. Mandelbaum \& C.R. Diniz. 1995. Characterization of a hemorrhagic factor, LHF-1, isolated from the Bushmaster snake (Lachesis muta muta) venom. Toxicon 33: 1653-1667.

Santoro, M.L. \& I. Sano Martins. 1993. Different Clotting mechanisms of Bothrops jararaca snake venom on human and rabbit plasmas. Toxicon 31: 733-742.

Sasa, M. \& S. Vazquez. 2003. Snakebite envenomation in Costa Rica: a revision of incidence in the decade 1990-2000. Toxicon 41: 19-22. 
Segre, L., J.A. Dolab, R.F. Funes, E. de Titto, O.D Salomón, A.R. de Roodt, A.I. Haas \& S.I. García. 2000. Accidentes humanos por ofidios en Argentina. Rev. Bras. Toxicol. 13: 41.

Siles Villarroel, M., R. Rolim Rosa, F. Zelante \& R. Guidolin. 1978/79. Evidenciaçao em camundongos da soroneutralizacáo paraspecífica entre venenos e antivenenos botrópicos. Mem. Inst. Butantan 42: 337-344.

Silva Haad, J.S. 1980/1981. Accidentes humanos por las serpientes de los géneros Bothrops y Lachesis. Mem. Inst. Butantan 44: 403-423.
Theakston, R.D.G., M.J. Lloyd-Jones \& H. Reid. 1977. Micro-ELISA for detecting and assaying snake venom and venom-antibody. A. Lancet 24: 639-641.

Theakston, R.D.G. \& H.A. Reid. 1983. Development of simple standard assay procedures for the characterization of snake venoms. Bull. WHO 61: 949-956.

Vogt, A. 1985. El porqué, cuándo, cómo y dónde de los ofidios. AméricaLee, Buenos Aires. 703 p.

WHO. 1981. Progress in the Characterization of Venoms and Standardization of Antivenoms Offset Publication, World Health Organization, Ginebra, Suiza. 44 p. 
